\title{
Enhanced and Fast Face Recognition by Hashing Algorithm
}

\author{
M. Sharif*, K. Ayub, D.Sattar, M. Raza, S. Mohsin \\ Department of Computer Science, \\ COMSATS Institute of Information \\ Technology Wah Cantt Pakistan. \\ *muhammadsharifmalik@yahoo.com
}

\begin{abstract}
This paper presents a face hashing technique for fast face recognition. The proposed technique employs the two existing algorithms, i.e., 2-D discrete cosine transformation and K-means clustering. The image has to go through different pre-processing phases and the two above-mentioned algorithms must be used in order to obtain the hash value of the face image. The searching process is increased by introducing a modified form of binary search. A new database architecture called Facebases has also been introduced to further speedup the searching process.
\end{abstract}

Keywords: DCT, K-means, Facebases, database, hashing, recognition.

\section{Introduction}

There are two basic concerns in face recognition systems namely face identification and face authentication [1][2][3][4]. For face identification, a system has to determine whether an input image matches a person's data in the database. For authentication, a system has to make sure that an input image has an exact match with a particular individual's data in the database records.

A typical face recognition system has generally three modules, i.e., preprocessing, feature selection criteria and classification module. The preprocessing step includes the segmentation process for finding face data in an image. The area of interest, the face in this case, is extracted in this step and normalization is performed on it. Sometimes, a preprocessing step also includes the feature extraction from the segmented face data like eyes, nose, lips, etc.

Several face recognition techniques have been proposed and developed based on extracting facial features and calculating geometrical distances in facial parts including distance from nose to eyes, distance from nose to forehead, distance between eyes, etc. Unfortunately, these techniques are not able to maintain the accuracy rate in recognizing facial parts. Moreover, neural networks came in market to maximize the recognition rate by improving the training set of images.
The proposed technique is related to face hashing and this section tries to present the existing techniques

which are related and can sometimes be helpful to face hashing. The idea of face hashing has been extracted from early graphical feature mapping techniques. For example, Noor Zaman et al. [5] presented the concept of face bar code in their paper but the problem is that they did not provide enough results to prove the validity of the technique.

V.V. Starovoitov et al. [6] in their paper described facial recognition using geometric features. In this technique the face is marked as different segments. These segments are created by marking different points on the face, as a result of which fifteen segments and twenty eight features of face are created between the points. These features are then measured by the distances between the points. Feng Yang et al. [7] used the geometrical mapping on the face which helped in pose variation and face identification. King Hong Cheung et al. [8] introduced the secrets of face hashing by using false assumption. There are a number of face recognition systems available for commercial use namely Cognitec [9], Eyematic [10], Viisage [11], Identix [12],[20], [21] and [13], among others. 


\section{Proposed algorithm}

The proposed technique is based on calculating numerical values of a face image for fast recognition. These values should be unique for each face in the database. For this purpose, 2-D Discrete Cosine Transformation (2-D DCT) has been selected for creating the face image. The calculated hash value of the face is then saved into the database with the corresponding face ID. Storing the database with respect to hash values will help to apply modified binary search to recognize the faces from the database. To further decrease the searching time, the database containing the hash values is separated into ten files (i.e. $0,1,2 \ldots 9$ ) corresponding to the first character of hash values. The whole process consists of two different phases: database generation phase and binary search for face recognition phase.

\subsection{Database Generation Phase}

The training database for images consists of two things, i.e., the pre-processed or normalized images and the database files having information about the saved images. The images to be stored in the database must follow the preprocessing step. Figure 1 depicts this scenario.

\subsubsection{The input Images}

A number of train face images were used for each individual to be stored in the database. Each train image for a person is different depending upon the conditions like pose, illumination, background and other facial expressions, among others. This will help to find the faces more accurately. All the images in the database should be in grayscale hence the very first step after image acquisition is to convert the image to grayscale (See Figure 2).

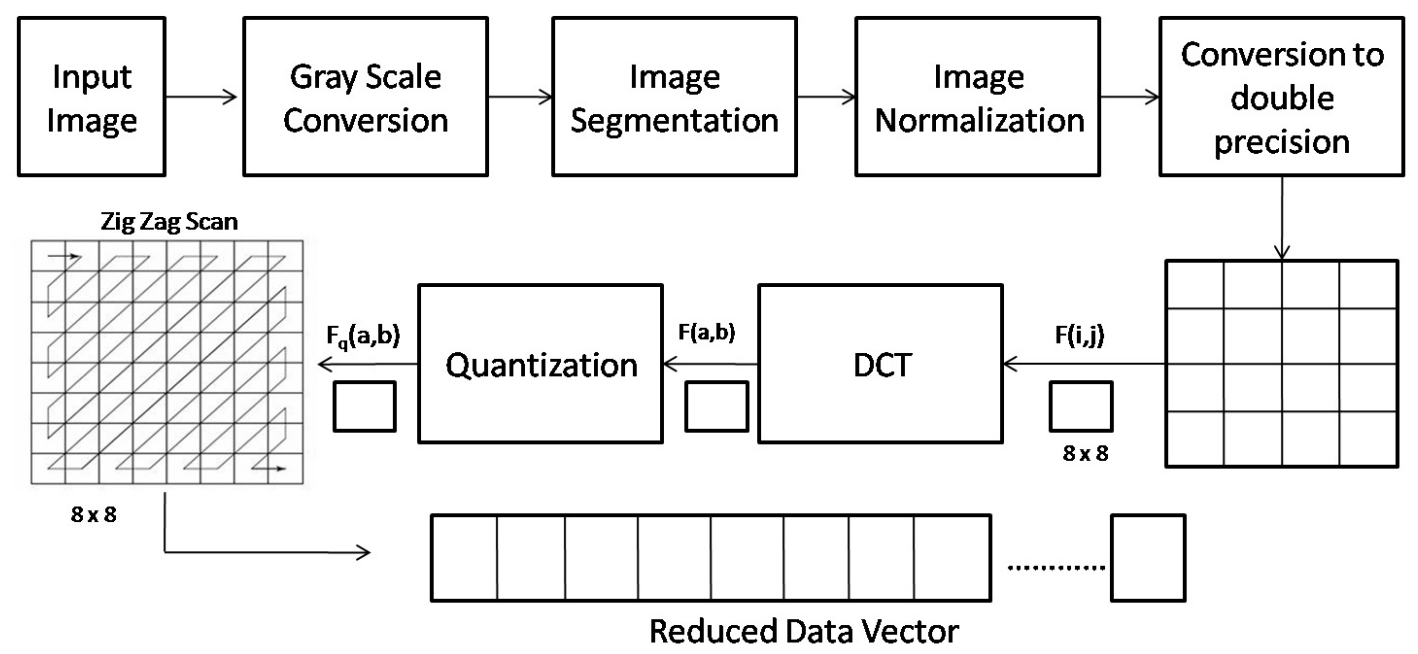

Figure1. Pre-processing and dimensionality reduction.

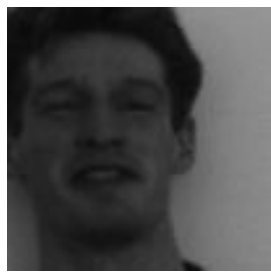

(a)

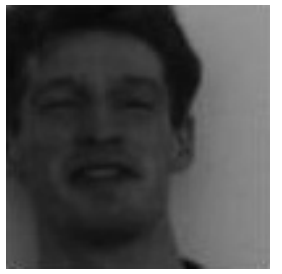

(b)

Figure 2. a) original image, b) grayscale image. 


\subsubsection{Preprocessing}

The preprocessing phase segments the face region from the input image. The face region is then normalized (scaling, sizing and positioning) in standard format of the stored database. The normalized image is then converted to double precision image intensity.

\subsubsection{Data Reduction}

The segmented faces are processed further for block-based transformation. These faces are divided into many blocks in order to apply linear transformation to them. The block size may be $8 \times 8$ or $16 \times 16$ and lie in a sequence. The linear transformation provides energy compaction (data compression) and a well-known linear transformation 2-D DCT has been selected for this purpose which provides only $\mathrm{N}$ number of coefficients from the block of size MxM $(N<M x M)$.

Let, for example $M=8$, i.e., block size be $M x M$ which after applying DCT is represented by N, say $\mathrm{N}=8$. These eight coefficients are high energy components in that block and have low frequency. The DCT is represented by the following equation as mentioned in [14] [15]:

$$
y(k)=w(k) \sum_{n=1}^{N} x(n) \cos \frac{\pi(2 n-1)(k-1)}{2 N}, k=1, \ldots, N
$$

where $x_{n}$ is even around $n=0$ and even around $n=N-1$ :

$$
w(k)=\left\{\begin{array}{c}
\frac{1}{N}, k=1 \\
\sqrt{\frac{2}{N}}, 2 \leq k \leq N
\end{array}\right.
$$

\subsubsection{Facebases Generation}

The vectors created using DCT coefficients are then quantized to discard the non significant information from the image (quantization is lossy) [14] [15]. These coefficients then pass to the next step for Facebase generation.
This paper introduces a term named Facebase for face database. The architecture of the proposed database (Facebases) is different from the traditional face databases in that it consists of ten database files, i.e., FB0, FB1, FB2, FB3... FB9 depending upon the first digit of the hash value (described later). This means that information of a face having the hash value starting with digit 0 will be saved into the database file FBO, information of a face having the hash value starting with digit 1 will be saved into the database file FB1 and so on.

The design of the Facebase generator uses the Kmeans clustering [16] algorithm for generation of Facebases from the training set of vectors. For this, the mean vector is calculated from all the reduced data vectors formed from the data reduction step described previously. The K-means filter [16] is then applied to the resultant vector with $\mathrm{k}$ clusters. The proposed technique selects four clusters which can be varied [16].

$$
\arg _{s} \min \sum_{i=1}^{k} \sum_{x_{j} \in s_{i}} \square x_{j}-\mu_{i} \square
$$

where $\left(\mathbf{x}_{1}, \mathbf{x}_{2}, \ldots, \mathbf{x}_{n}\right)$ is a set of vectors from the previous step.

$\mathrm{k}=$ number of clusters (partitions)

$\mathbf{S}=$ k sets i.e., $\mathbf{S}=\left\{S_{1}, S_{2} \ldots S_{k}\right\}$

Depending upon the four partitions, the four centroid values are calculated. Figure 3 shows four centroid values of a face image produced after applying the 2-D DCT transform, zig-zag scanning and the Facebase generation algorithm (through $\mathrm{K}$-means clustering)

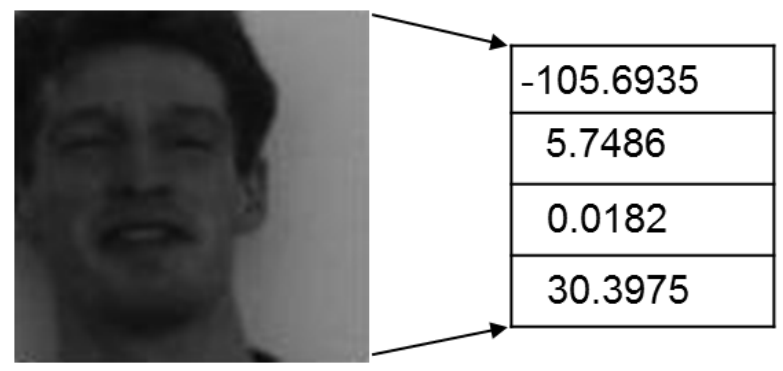

Figure 3. Four centroid values of a face image. 
The sum of these four centroid values is calculated and considered as the face hash value. See Figure 4.

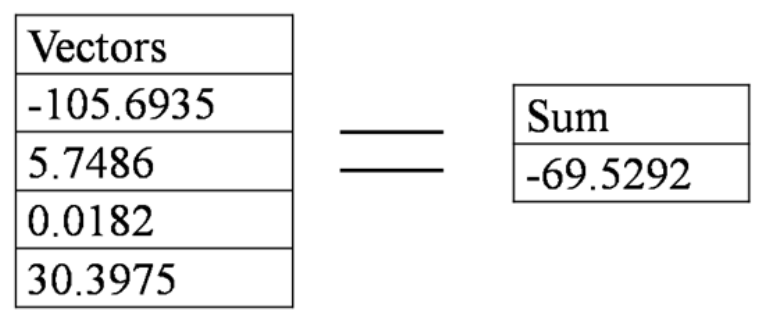

Figure 4. A Face hash.
The face hash value is then saved into the Facebases corresponding to the first digit of hash value (i.e., the sum of vectors is calculated and based on its first digit the corresponding Facebase is loaded for searching). Figure 4 shows that face value -69.5292 will be stored in FB6 (Facebase number 6 ). The whole face hashing process is explained in Figure 5.

The hashes in Facebases are stored in sorted order so that the modified binary search could be applied for fast searching. Figure 6 shows the format of a Facebase database.
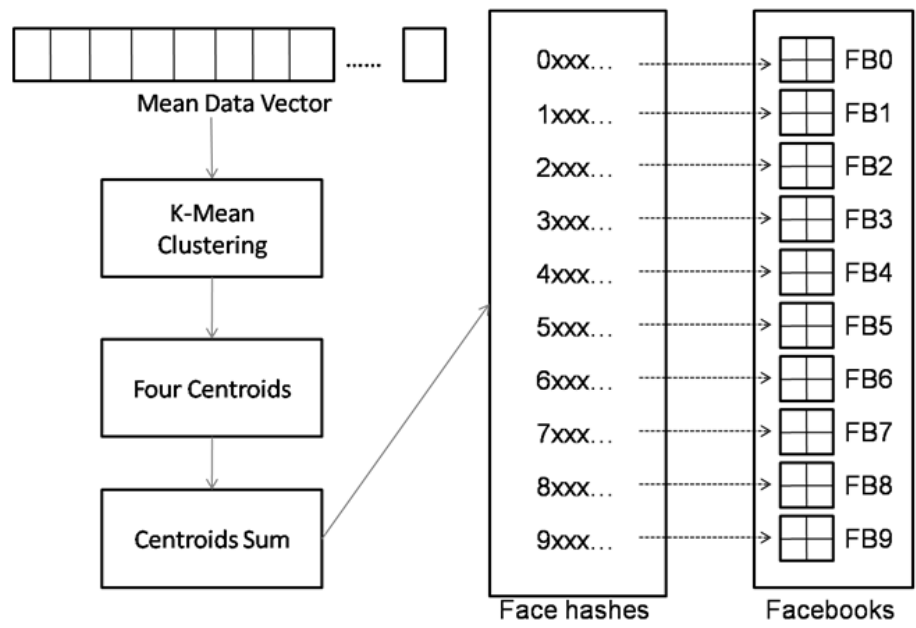

Figure 5. Face hash storing process.

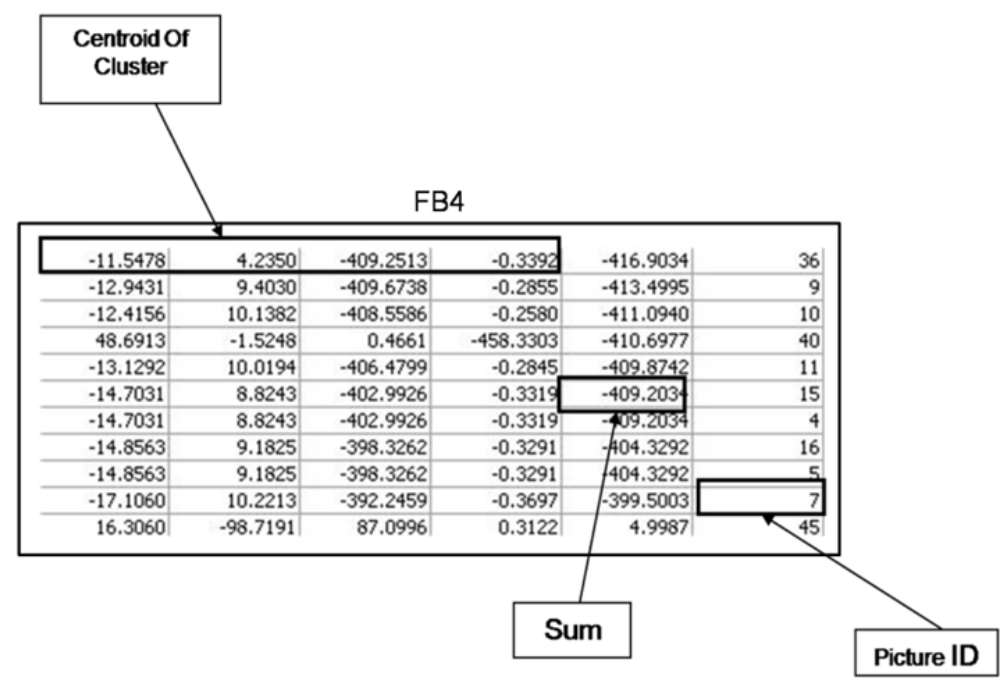

Figure 5. Figure 6. A Facebase database. 
Each entry in a database consists of six fields: four fields for the centroid of clusters (hash value), one field for the sum of centroids and one for the picture ID to keep track of pictures in the database. This proposed scheme of database arrangement stores the data in a symmetric and arranged order. The fields defined for each entry in a Facebase helps to track pictures as well as the hash values of each entry; therefore, it efficiently helps to speed up the recognition process because of requiring less searching time to find a match for test image in a database.

\subsubsection{Face Recognition Process}

The proposed scheme of Facebase generation and database record management helps to speed up the recognition rate and achieves the goal of automatic face recognition; it also makes the search fast and reduces bandwidth consumption. The initial steps followed in the proposed architecture for the recognition module are the same as in the database generation step i.e.:

\section{Proposed Algorithm: Face Recognition}

Take a segmented face region as an input image.

Convert the image to grayscale.

Segment the image and extract the face region.

Normalize the image.

Convert the image to double precision.

Apply 2-D DCT on the image.

Apply quantization.

Apply zig-zag scan to form the reduced data vector.

Apply the K-means clustering algorithm with $k=4$ and find the centroid values.

Sum the centroid values resulting in the face hash value and find the first digit of this hash value.

Depending upon the first digit, select the corresponding Facebase

Apply modified binary search to find the particular face ID in the selected Facebase. (Described later).

\subsection{The Modified Binary Search (MBS)}

To speed up the search process, a modified binary search algorithm has been introduced. As the hash values are calculated for images, there may be a difference of hash values depending upon illumination, pose, etc.; because of that, a threshold limit is introduced in modified binary search scheme. This limit may vary depending upon the database size (Total database entries in a Facebase)

The algorithm for MBS is as follows:

\section{Proposed Algorithm: MBS}

Check the length of the Facebase. (say N)

Check the number of entries in the Facebase with threshold limit $t$ (say $t=30$ )

if $(\mathrm{N}>\mathrm{t})$ then

Apply the correlation matching or minimum distance between vectors using the following equation:

$$
\gamma_{k}=\sum_{i=1}^{N}|| \alpha_{i}|-| \beta \|, k=1,2,3,4
$$

Now, calculate the sum of vectors :

$$
\theta=\gamma_{1}+\gamma_{2}+\gamma_{3}+\gamma_{4}
$$

or

$$
\theta=\sum_{i=1}^{4} \gamma_{i}
$$

where, $\boldsymbol{\alpha}$ represents the vectors (centroid) stored in Facebase $\boldsymbol{\beta}$ represents the new vectors of the new image $\gamma$ represents the absolute distance between stored and new vectors $\boldsymbol{\theta}$ represents the sum of absolute differences of these vectors $N$ represents the Facebase length Check for the entry whose correlation value results greater than or equal to 0.98 , otherwise the input image match will not exist in a database.

$$
\text { if }(\mathrm{N}>\mathrm{t}) \text { then }
$$

Check the first and last entry of Facebase ( first entry as $F_{b 1}$ and last entry as $F_{b L}$ )

If $(\mathrm{Fs}<\mathrm{Fb} 1)$, then (where Fs is the hash value to be searched and $\mathrm{Fb} 1$ is the first entry of the current Facebase) divide the threshold value by its half (say $t / 2=15$ ) and match the hash value with the 
last $t / 2$ entries from the previous Facebase of the selected one, i.e., Fs $€ \mathrm{Fb}-1$ [(t/2) L]

If $\left(F s>F b_{L}\right)$, (where the hash value to be searched is greater than the first entry of selected Facebase), divide the threshold value by its half (say $t / 2=15$ ) and match the hash value with the first $t / 2$ entries from the next Facebase of the selected one.

$\mathrm{Fs} \in \mathrm{Fb}+1[(\mathrm{t} / 2) 1]$

If $(F s \in F b L)$, (the hash value to be searched is within the first and last entry of the Facebase), it means that the match can take place in the selected Facebase. Now apply the normal binary search algorithm and find the closest match.
After finding the closest hash value, select the previous $t / 2$ values and the next $t / 2$ values of the image with closest hash value in the selected Facebase (call it the threshold limit values).

Apply the correlation matching on these values ( step 2, Equation 3)

Now, calculate the sum of vectors ( step 2, Equations 4 and 5)

Check for the entry whose correlation value results greater than or equal to 0.98 , otherwise the input image match will not exist in a database.

Figure 7 shows the way the modified binary search works.

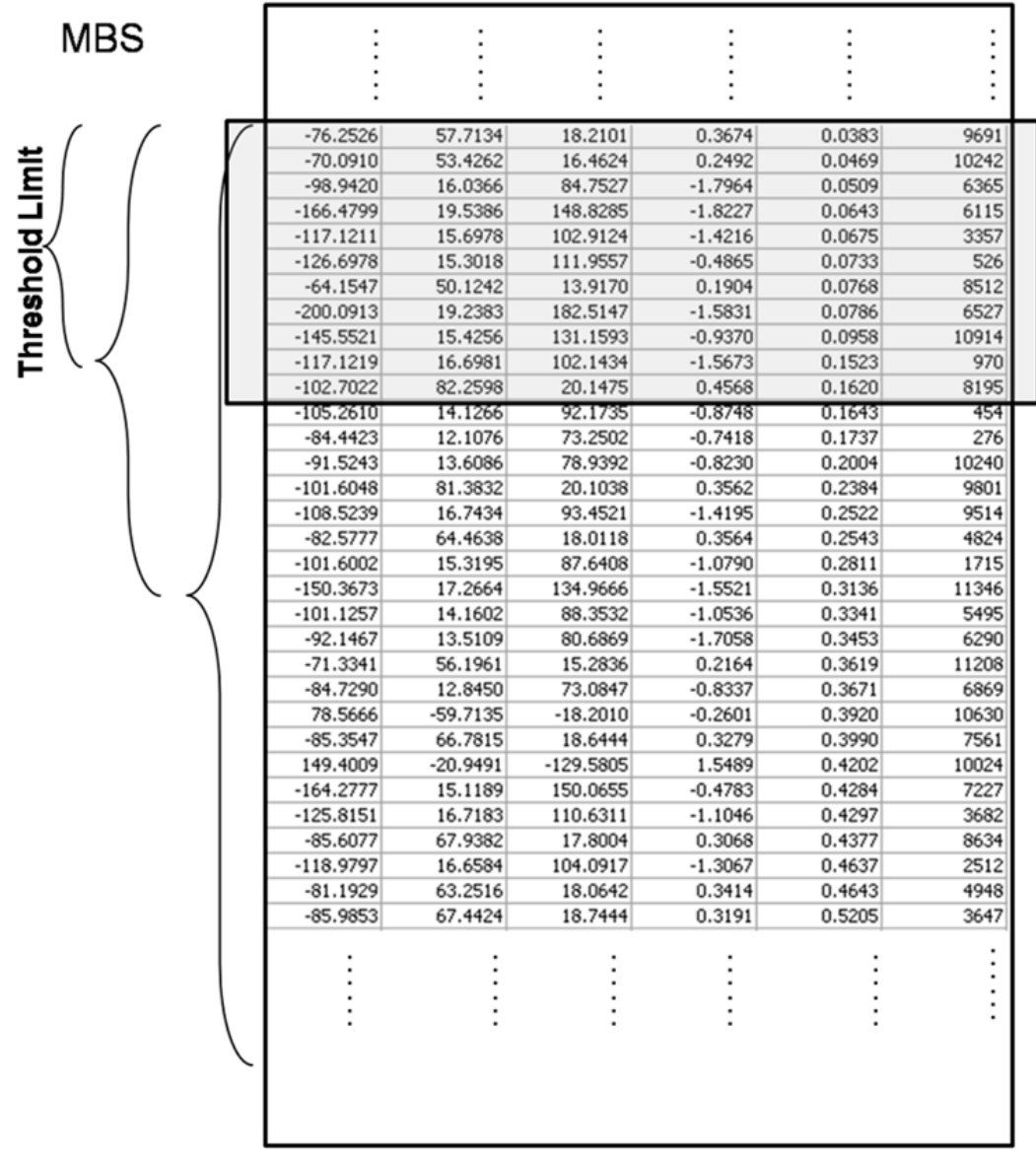

Figure 7. Modified binary search.
Search the test face in all the value within the threshold limit 


\section{Experiments and results}

\subsection{Results for the ORL Database}

The accuracy and performance of the existing symbolic LDA (linear discriminant analysis) is reviewed in the atomizing face recognition process using the "database of faces" (formerly 'The ORL database of faces'). The ORL face database [17] consists of 400 images in such a way that it contains ten images for each of the 40 unique persons in various poses. All the images have a uniform background with the persons standing in frontal position. The spatial and gray level intensities of the images are 92112 and 256 , respectively. In our test, all images are resized to $80 \times 80$. All the 400 images from the ORL database are used to assess the face recognition performance of the proposed scheme. Six images are randomly selected from the ten images of each person for training and the remaining is used for testing. Table 1 shows the training time and recognition rate comparison of the existing and the proposed techniques using the Yale face database.

The graph in Figure 8 shows the comparison of different techniques with respect to the time required for the training of images. The $x$-axis shows various techniques and the $y$-axis represents the training time in seconds.

\begin{tabular}{|c|c|c|c|}
\hline Methods & $\begin{array}{c}\text { Training Time } \\
\text { (Sec) }\end{array}$ & Resolution & Recognition Rate (\%) \\
\hline Fisherfaces & 98 & $80 \times 80$ & 92.8 \\
\hline Eigenfaces & 102 & $80 \times 80$ & 87.65 \\
\hline Symbolic PCA & 38 & $80 \times 80$ & 94.85 \\
\hline Symbolic ICA & 87 & $80 \times 80$ & 89.15 \\
\hline Symbolic KPCA & 110 & $80 \times 80$ & 95.45 \\
\hline Symbolic LDA & 19 & $80 \times 80$ & 97.5 \\
\hline Proposed & 25 & $80 \times 80$ & 98.2 \\
\hline
\end{tabular}

Table 1. Comparison of the results for the ORL Database [18].

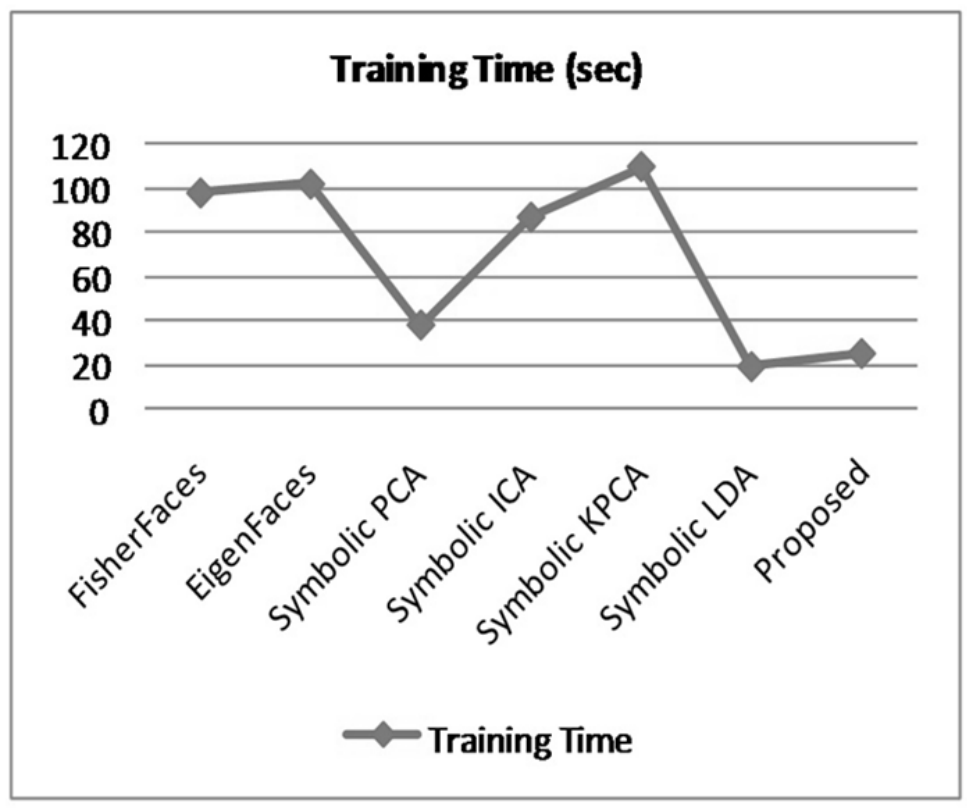

Figure 8. Training time comparison for the ORL database. 
Figure 9 shows the comparison of the recognition rate of various previously defined recognition techniques with the proposed scheme of recognition. The $y$-axis represents the recognition rate in percentage.

\subsection{Yale Face Database results}

The experiments are performed using the Yale database [19] to compare the face recognition accuracy of the proposed technique with other existing techniques. In these experiments, 9 images are arbitrarily taken from each class for training and the remaining is used for recognition or testing purpose. Table 2 shows the training time and recognition rate comparison of the existing and the proposed techniques using the Yale Face Database.

Figure 10 shows the comparison of different techniques with respect to the time required for training of images. The $\mathrm{x}$-axis shows various techniques and the $y$-axis represents the training time in seconds.

Figure 11 shows the comparison of the recognition rate of various previously defined recognition techniques with the proposed scheme of recognition. The $y$-axis represents the recognition rate in percentage.

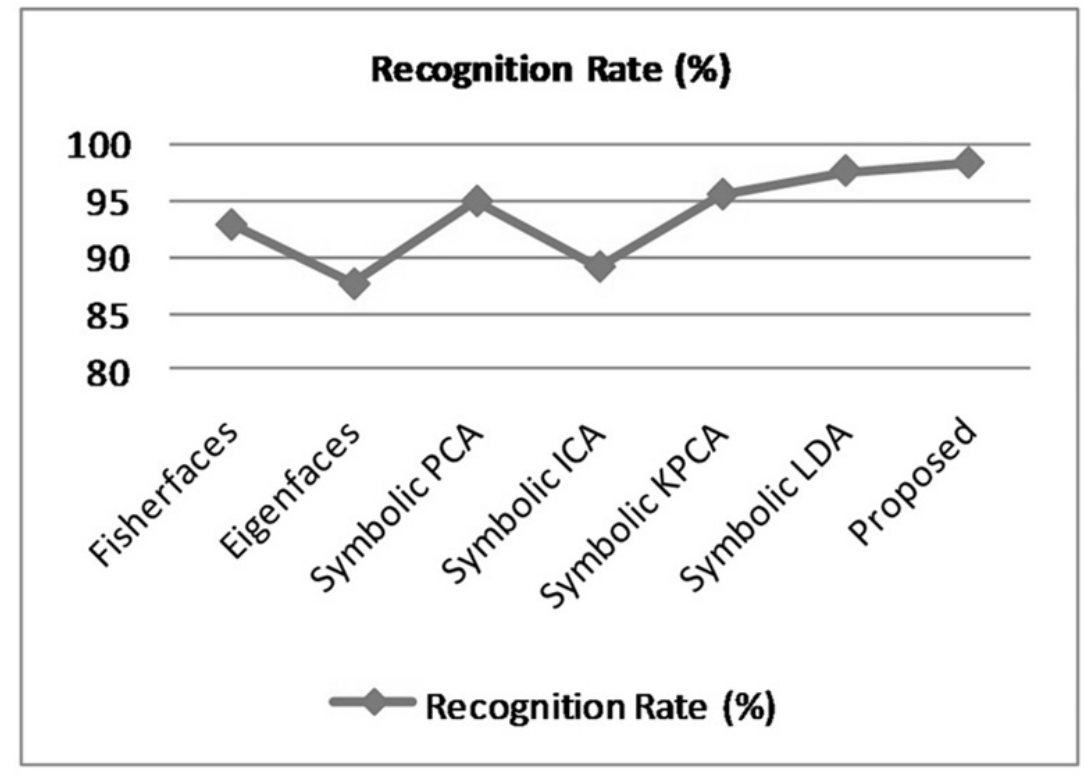

Figure 9. Accuracy comparison of different techniques for the ORL database.

\begin{tabular}{|c|c|c|c|}
\hline Methods & Training Time (Sec) & Resolution & Recognition Rate (\%) \\
\hline Fisherfaces & 59 & $128 \times 156$ & 89.85 \\
\hline Eigenfaces & 85 & $128 \times 156$ & 82.04 \\
\hline Symbolic PCA & 35 & $128 \times 156$ & 91.15 \\
\hline Symbolic ICA & 43 & $128 \times 156$ & 92.00 \\
\hline Symbolic KPCA & 98 & $128 \times 156$ & 94.55 \\
\hline Symbolic LDA & 18 & $128 \times 156$ & 96.15 \\
\hline Proposed & 31 & $128 \times 156$ & 96.66 \\
\hline
\end{tabular}

Table 2. Comparison of the Yale database results [18]. 


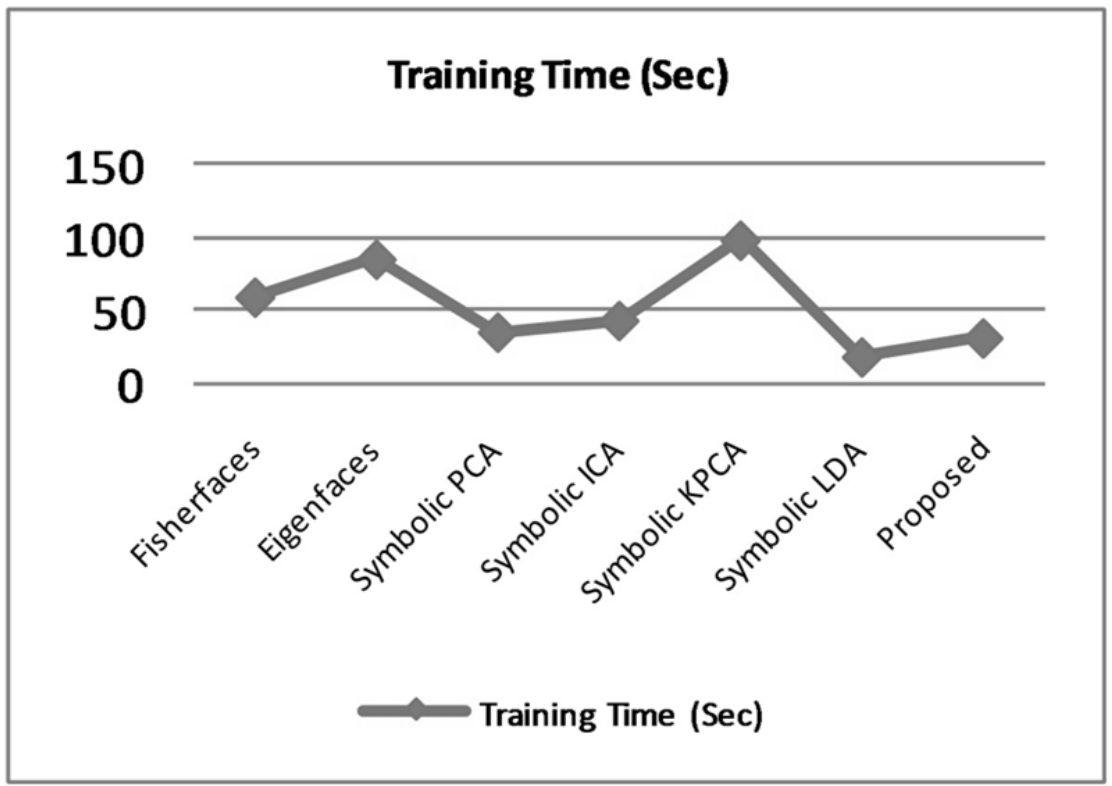

Figure 10. Training time comparison for the Yale database.

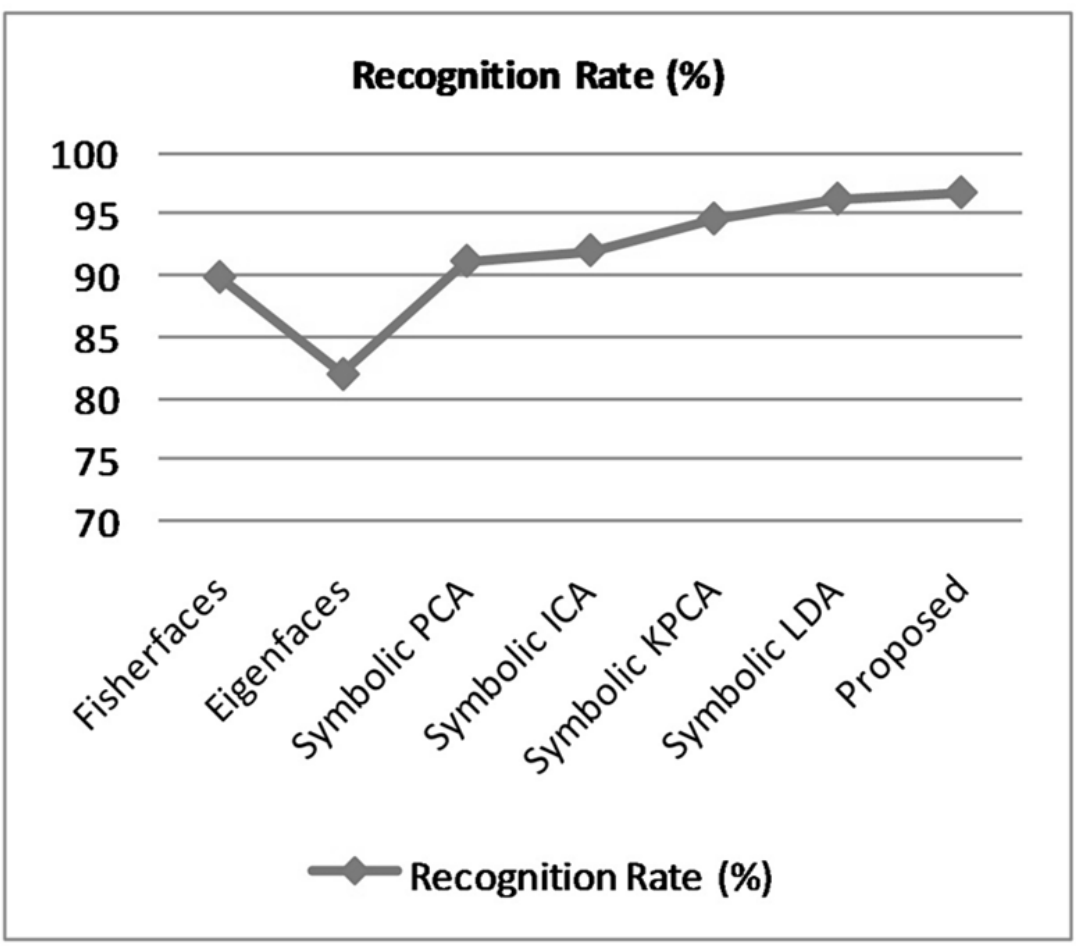

Figure 11. Recognition rate comparison of different techniques for the Yale database. 


\subsection{Recognition Time Analysis}

The recognition time analysis of the proposed system is given in Table 3 . For this purpose, a large database of 11,152 pictures was created containing the pictures from the ORL and Yale databases. Some pictures of different resolutions and backgrounds are also added to the database. The purpose of using different types of pictures is to check the system performance in different conditions. To conduct the experiment, different images were selected randomly from the database as test images which then went through preprocessing and recognition phases. Some of the results are shown in Table 3 . The average time found for face recognition is approximately 142 milliseconds ( 0.14 seconds per image).

\section{Conclusions and discussion}

Recognition in the proposed technique is achieved using the signal compression technique. Data for building a database are first compressed and then coded in the form of vectors; therefore, each individual's data are recorded in the form of numbers or vectors. There are a number of advantages of using compression techniques for recognition systems especially in bandwidth consumption problems. Firstly, an individual's face is decomposed into blocks and then processed by block-based transforms, i.e., the DCT transform. Then transformed data is coded and used for making vectors. Afterwards, the sum of these vectors is calculated which gives a hash value against each individual's entry. A number of such vectors from an individual's data constitute a Facebase of a particular individual. The efficient use of the block-based transform reduces the original data to high energy coefficients which are very few in number as compared with the original data. Making a hash value reduces the searching time efficiently and maximizes the recognition rate.

The main purpose of the proposed technique is fast face recognition so that it can be helpful to recognize a face in a large database of faces. The technique is focused on both searching time and recognition accuracy. On the basis of the experiments and the observed results, it has been shown that the proposed algorithm reduces both the search time required by the recognition algorithm and the training time required. This algorithm can be used in the future in the areas in which fast and rapid recognition is needed.

\begin{tabular}{|c|c|c|c|c|c|c|}
\hline Pic ID & $\begin{array}{c}\text { Facebase } \\
\text { No }\end{array}$ & $\begin{array}{c}\text { Length of } \\
\text { FB }\end{array}$ & $\begin{array}{c}\text { Found at } \\
\text { Index }\end{array}$ & $\begin{array}{c}\text { Pre-Processing Time } \\
(\mathbf{m S e c})\end{array}$ & $\begin{array}{c}\text { Searching Time } \\
(\mathbf{m S e c})\end{array}$ & $\begin{array}{c}\text { Total Time } \\
(\mathbf{m S e c})\end{array}$ \\
\hline 1 & 1 & 3761 & 216 & 135 & 6.34 & 142 \\
\hline 100 & 1 & 3761 & 659 & 139 & 6.55 & 136 \\
\hline 1000 & 4 & 1015 & 303 & 129 & 8.67 & 137 \\
\hline 2000 & 3 & 1253 & 162 & 125 & 8.6 & 137 \\
\hline 4000 & 5 & 911 & 176 & 134 & 7.58 & 134 \\
\hline 5000 & 1 & 3761 & 2263 & 135 & 8.35 & 141 \\
\hline 7000 & 3 & 1253 & 432 & 136 & 7.06 & 143 \\
\hline 8000 & 1 & 3761 & 2884 & 127 & 8.94 & 143 \\
\hline 10000 & 2 & 1737 & 893 & 160 & 8.78 & 136 \\
\hline 11000 & 1 & 3761 & 2379 & 136 & & 169 \\
\hline 11152 & 7 & 741 & 258 & & & 142 \\
\hline
\end{tabular}

Table 3. Recognition time analysis Total Pictures $=11,152$.

Legend:

FB: Facebase

mSec: milliseconds 


\section{References}

[1] R. Chellappa, C.L. Wilson, and S. Sirohey, "Human and machine recognition of faces: A survey," Proc. IEEE, vol. 83, pp. 705-740, 1995.

[2] H. Wechsler, P. Phillips, V. Bruce, F. Soulie, and T. Huang, Face Recognition: From Theoryto Applications, Springer-Verlag, 1996.

[3] W. Zhao, R. Chellappa, A. Rosenfeld, and P.J. Phillips, "Face recognition: A literature survey", CVL Technical Report, University of Maryland, 2000.

[4] S. Gong, S.J. McKenna, and A. Psarrou, Dynamic Vision: from Images to Face Recognition, Imperial College Press and World Scientific Publishing, 2000.

[5] Noor Zaman, Abnan Alam Khan, Mansoor uz Zafar Dawood, Abdul Aziz, "Face Recognition Techniques (FRT) based on Face ratio under controlled conditions", IEEE Proceeding of International Symposium on Biometrics \& Security Techniques 2008. PP 3-8.

[6] V.V. Starovoitov, D.I Samal, D.V. Briliuk, "Three Approaches For Face Recognition", The 6-th International Conference on Pattern Recognition and Image Analysis October 21-26, 2002, Velikiy Novgorod, Russia, pp. 707-711.

[7] Feng Yang and Adam Krzyżak,"Face Recognition under Significant Pose Variation", Canadian Conference (IEEE), Electrical and Computer Engineering, 2007. CCECE 2007 page(s): 1313-1316.

[8]K.-H. Cheung, A. Kong, D. Zhang, M. Kamel and J. You. Revealing the secret of Face Hashing in Intl. Conf. on Biometrics, volume 3832 of LNCS, pages 106-112, 2006.

[9] Carlos Gomez-Rodriguez, David Weir, John Carroll, "Parsing mildly non-projective dependency structures", Cognitive science research papers, US university of Sussex.

[10] Trevor Darrell, Neal Checka, Alice Oh and Louis Phillipe Morency, "Exploring Vision Based Interfaces: How to use Your head in Dual Pointing Tasks", Al Memo 2002-001 January 2002.

[11] Junwen Wu; Trivedi, M.M, "A Binary Tree for Probability Learning in Eye Detection", Computer Vision and Pattern Recognition, 2005 IEEE Computer Society Conference on 25-25 June 2005 Page(s):170 - 170.
[12] Moisture Discriminating optics, "Real world advantages of Moisture Discriminating optics in live scan digital finger print imaging", issued October 23, 2002 identix incorporated.

[13] Qinfeng Shi, Hanxi Li, Chunhua Shen, Rapid Face Recognition Using Hashing, In IEEE Computer Society Conference on Computer Vision and Pattern Recognition (CVPR 10), San Francisco, USA, June 1318,2010

[14] Jain, A.K. Fundamentals of Digital Image Processing, Englewood Cliffs, NJ: Prentice-Hall, 1989.

[15] Pennebaker, W.B., and J.L. Mitchell. JPEG Still Image Data Compression Standard, New York, NY: Van Nostrand Reinhold, 1993. Chapter 4.

[16] Hartigan, J. A. (1975). Clustering Algorithms. Wiley. MR0405726. ISBN 0-471-35645-X.

[17] "AT\&T "The Database of Faces" (formerly "The ORL Database of Faces")".

[18] P.S. Hiremath and Prabhakar C.J.," Face Recognition Technique Using Symbolic Linear Discriminant Analysis Method", P. Kalra and S. Peleg (Eds.): ICVGIP 2006, LNCS 4338, pp. 641 - 649, 2006.

[19] The Yale Face Database, http://cvc.yale.edu/projects/yalefaces/yalefaces.html, Last visited on 28th July, 2009.

[20] David C. L. Ngo, Andrew B. J. Teoh and Alwyn Goh, "Eigenspace-Based Face Hashing.", Biometric Authentication, First International Conference, ICBA 2004, Hong Kong, China, July 15-17, 2004, Proceedings 2004.

[21] Ngo, D.C.L.; Teoh, A.B.J.; Goh, A., "Biometric Hash: High-Confidence Face Recognition" IEEE Transactions on Circuits and Systems for Video Technology, June 2006, Volume: 16 Issue: 6, page(s): $771-775$ 